\title{
A Tverberg Type Theorem for Matroids
}

\author{
Imre Bárány $\quad$ Gil Kalai Roy Meshulam
}

April 19, 2022

\begin{abstract}
Let $b(M)$ denote the maximal number of disjoint bases in a matroid $M$. It is shown that if $M$ is a matroid of rank $d+1$, then for any continuous map $f$ from the matroidal complex $M$ into $\mathbb{R}^{d}$ there exist $t \geq \sqrt{b(M)} / 4$ disjoint independent sets $\sigma_{1}, \ldots, \sigma_{t} \in M$ such that $\bigcap_{i=1}^{t} f\left(\sigma_{i}\right) \neq \emptyset$.
\end{abstract}

\section{Introduction}

Tverberg's theorem [15] asserts that if $V \subset \mathbb{R}^{d}$ satisfies $|V| \geq(k-1)(d+1)+1$, then there exists a partition $V=V_{1} \cup \cdots \cup V_{k}$ such that $\bigcap_{i=1}^{k} \operatorname{conv}\left(V_{i}\right) \neq \emptyset$. Tverberg's theorem and some of its extensions may be viewed in the following general context. For a simplicial complex $X$ and $d \geq 1$, let the affine Tverberg number $T(X, d)$ be the maximal $t$ such that for any affine map $f: X \rightarrow \mathbb{R}^{d}$, there exist disjoint simplices $\sigma_{1}, \ldots, \sigma_{t} \in X$ such that $\cap_{i=1}^{t} f\left(\sigma_{i}\right) \neq \emptyset$. The topological Tverberg number $T T(X, d)$ is defined similarly where now $f: X \rightarrow \mathbb{R}^{d}$ can be an arbitrary continuous map.

Let $\Delta_{n}$ denote the $n$-simplex and let $\Delta_{n}^{(d)}$ be its $d$-skeleton. Using the above terminology, Tverberg's theorem is equivalent to $T\left(\Delta_{(k-1)(d+1)}, d\right)=k$ which is clearly the same as $T\left(\Delta_{(k-1)(d+1)}^{(d)}, d\right)=k$. Similarly, the topological Tverberg theorem of Bárány, Shlosman and Szücs [2] states that if $p$ is prime then $T T\left(\Delta_{(p-1)(d+1)}, d\right)=p$. Schöneborn and Ziegler [14] proved that this implies the stronger statement $T T\left(\Delta_{(p-1)(d+1)}^{(d)}, d\right)=p$. This result was extended by Özaydin [13] for the case when $p$ is a prime power. The question whether the topological Tverberg theorem holds for every $\mathrm{p}$ that is not a prime power had been open for long. Very recently, and quite surprisingly, Frick [7] has constructed a counterexample for every non-prime power $p$. His construction is built on work by Mabillard and Wagner [10]. See also [4] and [1] for further counterexamples.

There is a colourful version of Tverberg theorem. To state it let $n=r(d+1)-1$ and assume that the vertex set $V$ of $\Delta_{n}$ is partitioned into $d+1$ classes (called colours) and that each colour class contains exactly $r$ vertices. We define $Y_{r, d}$ as the subcomplex of $\Delta_{n}$ (or $\left.\Delta_{n}^{(d)}\right)$ consisting of those $\sigma \subset V$ that contain at most one vertex from each colour class. The colourful Tverberg theorem of Živaljević and Vrećica [16] asserts that $T T\left(Y_{2 p-1, d}, d\right) \geq p$ for prime $p$ which implies that $T T\left(Y_{4 k-1, d}, d\right) \geq k$ for arbitrary $k$. A neat and more recent theorem of Blagojević, Matschke, and Ziegler [5] says that $T T\left(Y_{r, d}, d\right)=r$ if $r+1$ is a prime, which is clearly best possible. Further information on Tverberg's theorem can be found in Matoušek's excellent book [12]. 
Let $M$ be a matroid (possibly with loops) with rank function $\rho$ on the set $V$. We identify $M$ with the simplicial complex on $V$ whose simplices are the independent sets of $M$. It is well known (see e.g. Theorem 7.8.1 in [3]) that $M$ is $(\rho(V)-2)$-connected. Note that both $\Delta_{n}^{(d)}$ and $Y_{r, d}$ are matroids of rank $d+1$. In this note we are interested in bounding $T T(M, d)$ for a general matroidal complex $M$. Let $b(M)$ denote the maximal number of pairwise disjoint bases in $M$. Our main result is the following

Theorem 1. Let $M$ be a matroid of rank $d+1$. Then

$$
T T(M, d) \geq \sqrt{b(M)} / 4 .
$$

In Section 2 we give a lower bound on the topological connectivity of the deleted join of matroids. In Section 3 we use this bound and the approach of [2, 16] to prove Theorem 1,

\section{Connectivity of Deleted Joins of Matroids}

We recall some definitions. For a simplicial complex $Y$ on a set $V$ and an element $v \in V$ such that $\{v\} \in Y$, denote the star and link of $v$ in $Y$ by

$$
\begin{aligned}
& \operatorname{st}(Y, v)=\{\sigma \subset V:\{v\} \cup \sigma \in Y\} \\
& \operatorname{lk}(Y, v)=\{\sigma \in \operatorname{st}(Y, v): v \notin \sigma\} .
\end{aligned}
$$

For a subset $V^{\prime} \subset V$ let $Y\left[V^{\prime}\right]=\left\{\sigma \subset V^{\prime}: \sigma \in Y\right\}$ be the induced complex on $V^{\prime}$. We regard $\operatorname{st}(Y, v), \operatorname{lk}(Y, v)$ and $Y\left[V^{\prime}\right]$ as complexes on the original set $V$ (keeping in mind that not all elements of $V$ have to be vertices of these complexes). Let $f_{i}(Y)$ denote the number of $i$-simplices in $Y$. Let $X_{1}, \ldots, X_{k}$ be simplicial complexes on the same set $V$ and let $V_{1}, \ldots, V_{k}$ be $k$ disjoint copies of $V$ with bijections $\pi_{i}: V \rightarrow V_{i}$. The join $X_{1} * \cdots * X_{k}$ is the simplicial complex on $\bigcup_{i=1}^{k} V_{i}$ with simplices $\bigcup_{i=1}^{k} \pi_{i}\left(\sigma_{i}\right)$ where $\sigma_{i} \in X_{i}$. The deleted join $\left(X_{1} * \cdots * X_{k}\right)_{\Delta}$ is the subcomplex of the join consisting of all simplices $\bigcup_{i=1}^{k} \pi_{i}\left(\sigma_{i}\right)$ such that $\sigma_{i} \cap \sigma_{j}=\emptyset$ for $1 \leq i \neq j \leq k$. When all $X_{i}$ are equal to $X$, we denote their deleted join by $X_{\Delta}^{* k}$. Note that $\mathbb{Z}_{k}$ acts freely on $X_{\Delta}^{* k}$ by cyclic shifts.

Claim 2. Let $M_{1}, \ldots, M_{k}$ be matroids on the same set $V$, with rank functions $\rho_{1}, \ldots, \rho_{k}$. Suppose $A_{1}, \ldots, A_{k}$ are disjoint subsets of $V$ such that $A_{i}$ is a union of at most $m$ independent sets in $M_{i}$. Then $Y=\left(M_{1} * \cdots * M_{k}\right)_{\Delta}$ is $\left(\left\lceil\frac{1}{m+1} \sum_{i=1}^{k}\left|A_{i}\right|\right\rceil-2\right)$-connected.

Proof: Let $c=\left\lceil\frac{1}{m+1} \sum_{i=1}^{k}\left|A_{i}\right|\right\rceil-2$. If $k=1$ then $\rho_{1}(V) \geq\left\lceil\frac{\left|A_{1}\right|}{m}\right\rceil$ and hence $Y=M_{1}$ is $\left(\left\lceil\frac{\left|A_{1}\right|}{m}\right\rceil-2\right)$-connected. For $k \geq 2$ we establish the Claim by induction on $f_{0}(Y)=$ $\sum_{i=1}^{k} f_{0}\left(M_{i}\right)$. If $f_{0}(Y)=0$ then all $A_{i}$ 's are empty and the Claim holds. We henceforth assume that $f_{0}(Y)>0$ and consider two cases:

a) If $M_{i}=M_{i}\left[A_{i}\right]$ for all $1 \leq i \leq k$ then $Y=M_{1} * \cdots * M_{k}$ is a matroid of rank

$$
\sum_{i=1}^{k} \rho_{i}(V) \geq \sum_{i=1}^{k}\left\lceil\frac{\left|A_{i}\right|}{m}\right\rceil \geq\left\lceil\frac{\sum_{i=1}^{k}\left|A_{i}\right|}{m}\right\rceil .
$$

Hence $Y$ is $\left(\left\lceil\frac{\sum_{i=1}^{k}\left|A_{i}\right|}{m}\right\rceil-2\right)$-connected.

b) Otherwise there exists an $1 \leq i_{0} \leq k$ such that $M_{i_{0}} \neq M_{i_{0}}\left[A_{i_{0}}\right]$. Choose an element 
$v \in V-A_{i_{0}}$ such that $\{v\} \in M_{i_{0}}$. Without loss of generality we may assume that $i_{0}=1$ and that $v \notin \bigcup_{i=1}^{k-1} A_{i}$. Let $S=\bigcup_{i=1}^{k} V_{i}$ and let $Y_{1}=Y\left[S-\left\{\pi_{1}(v)\right\}\right], Y_{2}=\operatorname{st}\left(Y, \pi_{1}(v)\right)$. Then

$$
Y_{1}=\left(M_{1}[V-\{v\}] * M_{2} * \cdots * M_{k}\right)_{\Delta} .
$$

Noting that $f_{0}\left(Y_{1}\right)=f_{0}(Y)-1$ and applying the induction hypothesis to the matroids $M_{1}[V-\{v\}], M_{2}, \ldots, M_{k}$ and the sets $A_{1}, \ldots, A_{k}$, it follows that $Y_{1}$ is $c$-connected. We next consider the connectivity of $Y_{1} \cap Y_{2}$. Write $A_{1}=\bigcup_{j=1}^{t} C_{j}$ where $t \leq m, C_{j} \in M_{1}$ for all $1 \leq j \leq t$, and the $C_{j}$ 's are pairwise disjoint. Since $\{v\} \in M_{1}$, it follows that there exist $\left\{C_{j}^{\prime}\right\}_{j=1}^{t}$ such that $C_{j}^{\prime} \subset C_{j},\left|C_{j}^{\prime}\right| \geq\left|C_{j}\right|-1$, and $C_{j}^{\prime} \in \operatorname{lk}\left(M_{1}, v\right)$ for all $1 \leq j \leq t$. Let

$$
M_{i}^{\prime}= \begin{cases}\operatorname{lk}\left(M_{1}, v\right) & i=1 \\ M_{i}[V-\{v\}] & 2 \leq i \leq k\end{cases}
$$

and

$$
A_{i}^{\prime}= \begin{cases}\bigcup_{j=1}^{t} C_{j}^{\prime} & i=1 \\ A_{i} & 2 \leq i \leq k-1 \\ A_{k}-\{v\} & i=k\end{cases}
$$

Observe that

$$
Y_{1} \cap Y_{2}=\operatorname{lk}\left(Y, \pi_{1}(v)\right)=\left(M_{1}^{\prime} * \cdots * M_{k}^{\prime}\right)_{\Delta}
$$

and that $A_{i}^{\prime}$ is a union of at most $m$ independent sets in $M_{i}^{\prime}$ for all $1 \leq i \leq k$. Noting that $f_{0}\left(Y_{1} \cap Y_{2}\right) \leq f_{0}(Y)-1$ and applying the induction hypothesis to the matroids $M_{1}^{\prime}, \ldots, M_{k}^{\prime}$ and the sets $A_{1}^{\prime}, \ldots, A_{k}^{\prime}$, it follows that $Y_{1} \cap Y_{2}$ is $c^{\prime}$-connected where

$$
\begin{aligned}
c^{\prime} & =\left\lceil\frac{1}{m+1} \sum_{i=1}^{k}\left|A_{i}^{\prime}\right|\right]-2 \\
& =\left\lceil\frac{1}{m+1}\left(\sum_{j=1}^{t}\left|C_{j}^{\prime}\right|+\sum_{i=2}^{k-1}\left|A_{i}\right|+\left|A_{k}-\{v\}\right|\right)\right]-2 \\
& \geq\left\lceil\frac{1}{m+1}\left(\left|A_{1}\right|-m+\sum_{i=2}^{k-1}\left|A_{i}\right|+\left|A_{k}\right|-1\right)\right]-2=c-1 .
\end{aligned}
$$

As $Y_{1}$ is $c$-connected, $Y_{2}$ is contractible and $Y_{1} \cap Y_{2}$ is $(c-1)$-connected, it follows that $Y=Y_{1} \cup Y_{2}$ is $c$-connected.

Let $M$ be a matroid on $V$ with $b(M)=b$ disjoint bases $B_{1}, \ldots, B_{b}$. Let $I_{1} \cup \cdots \cup I_{k}$ be a partition of $[b]$ into almost equal parts $\left\lfloor\frac{b}{k}\right\rfloor \leq\left|I_{i}\right| \leq\left\lceil\frac{b}{k}\right\rceil$. Applying Claim 2 with $M_{1}=\cdots=M_{k}=M$ and $A_{i}=\cup_{j \in I_{i}} B_{j}$, we obtain:

Corollary 3. The connectivity of $M_{\Delta}^{* k}$ is at least

$$
\frac{b \rho(V)}{\left\lceil\frac{b}{k}\right\rceil+1}-2
$$

We suggest the following: 
Conjecture 4. For any $k \geq 1$ there exists an $f(k)$ such that if $b(M) \geq f(k)$ then $M_{\Delta}^{* k}$ is $(k \rho(V)-2)$-connected.

Remark: Let $M$ be the rank 1 matroid on $m$ points $M=\Delta_{m-1}^{(0)}$. The chessboard complex $C(k, m)$ is the $k$-fold deleted join $M_{\Delta}^{* k}$. Chessboard complexes play a key role in the works of Živaljević and Vrećica [16] and Blagojević, Matschke, and Ziegler [5] on the colourful Tverberg theorem. Let $k \geq 2$. Garst [9] and Živaljević and Vrećica [16] proved that $C(k, 2 k-1)$ is $(k-2)$-connected. On the other hand, Friedman and Hanlon [8] showed that $\tilde{H}_{k-2}(C(k, 2 k-2) ; \mathbb{Q}) \neq 0$, so $C(k, 2 k-2)$ is not $(k-2)$-connected. This implies that the function $f(k)$ in Conjecture 4 must satisfy $f(k) \geq 2 k-1$.

\section{A Tverberg Type Theorem for Matroids}

We recall some well-known topological facts (see [2]). For $m \geq 1, k \geq 2$ we identify the sphere $S^{m(k-1)-1}$ with the space

$$
\left\{\left(y_{1}, \ldots, y_{k}\right) \in\left(\mathbb{R}^{m}\right)^{k}: \sum_{i=1}^{k}\left|y_{i}\right|^{2}=1, \sum_{i=1}^{k} y_{i}=0 \in \mathbb{R}^{m}\right\} .
$$

The cyclic shift on this space defines a $\mathbb{Z}_{k}$ action on $S^{m(k-1)-1}$. The action is free for prime $k$.

The $k$-fold deleted product of a space $X$ is the $\mathbb{Z}_{k}$-space given by

$$
X_{D}^{k}=X^{k}-\left\{(x, \ldots, x) \in X^{k}: x \in X\right\} .
$$

For $m \geq 1$ define a $\mathbb{Z}_{k}$-map

$$
\phi_{m, k}:\left(\mathbb{R}^{m}\right)_{D}^{k} \rightarrow S^{m(k-1)-1}
$$

by

$$
\phi_{m, k}\left(x_{1}, \ldots, x_{k}\right)=\frac{\left(x_{1}-\frac{1}{k} \sum_{i=1}^{k} x_{i}, \ldots, x_{k}-\frac{1}{k} \sum_{i=1}^{k} x_{i}\right)}{\left(\sum_{j=1}^{k}\left|x_{j}-\frac{1}{k} \sum_{i=1}^{k} x_{i}\right|^{2}\right)^{1 / 2}} .
$$

We'll also need the following result of Dold [6] (see also Theorem 6.2.6 in [11]):

Theorem 5 (Dold). Let $p$ be a prime and suppose $X$ and $Y$ are free $\mathbb{Z}_{p}$-spaces such that $\operatorname{dim} Y=k$ and $X$ is $k$-connected. Then there does not exist a $\mathbb{Z}_{p}$-map from $X$ to $Y$.

Proof of Theorem 1; Let $M$ be a matroid on the vertex set $V$, and let $f: M \rightarrow \mathbb{R}^{d}$ be a continuous map. Let $b=b(M)$ and choose a prime $\sqrt{b} / 4 \leq p \leq \sqrt{b} / 2$. We'll show that there exist disjoint simplices (i.e. independent sets) $\sigma_{1}, \ldots, \sigma_{p} \in M$ such that $\bigcap_{i=1}^{p} f\left(\sigma_{i}\right) \neq \emptyset$. Suppose for contradiction that $\bigcap_{i=1}^{p} f\left(\sigma_{i}\right)=\emptyset$ for all such choices of $\sigma_{i}$ 's. Then $f$ induces a continuous $\mathbb{Z}_{p}$-map

$$
f_{*}: M_{\Delta}^{* p} \rightarrow\left(\mathbb{R}^{d+1}\right)_{D}^{p}
$$

as follows. If $x_{1}, \ldots, x_{p}$ have pairwise disjoint supports in $M$ and $\left(t_{1}, \ldots, t_{p}\right) \in \mathbb{R}_{+}^{p}$ satisfies $\sum_{i=1}^{p} t_{i}=1$ then

$$
f_{*}\left(t_{1} \pi_{1}\left(x_{1}\right)+\cdots+t_{p} \pi_{p}\left(x_{p}\right)\right)=\left(t_{1}, t_{1} f\left(x_{1}\right), \ldots, t_{p}, t_{p} f\left(x_{p}\right)\right) \in\left(\mathbb{R}^{d+1}\right)_{D}^{p} .
$$


Hence $\phi_{d+1, p} f_{*}$ is a $\mathbb{Z}_{p}$-map between the free $\mathbb{Z}_{p}$-spaces $M_{\Delta}^{* p}$ and $S^{(d+1)(p-1)-1}$. This however contradicts Dold's Theorem since by Corollary 3 the connectivity of $M_{\Delta}^{* p}$ is at least

$$
\frac{b(d+1)}{\left\lceil\frac{b}{p}\right\rceil+1}-2 \geq(d+1)(p-1)-1
$$

by the choice of $p$.

Acknowledgements. Research of Imre Bárány was partially supported by ERC advanced grant 267165, and by Hungarian National grant K 83767. Research of Gil Kalai was supported by ERC advanced grant 320924. Research of Roy Meshulam is supported by ISF and GIF grants.

\section{References}

[1] S. Avvakumov, I. Mabillard, A. Skopenkov, U. Wagner, Eliminating higher-multiplicity intersections, III. Codimension 2, (2015) 16 pages, arXiv:1511.03501

[2] I. Bárány, S. Shlosman and A. Szűcs, On a topological generalization of a theorem of Tverberg, J. London Math. Soc. 23(1981) 158-164.

[3] A. Björner, Topological methods. in Handbook of Combinatorics (R. Graham, M. Grötschel, and L. Lovász, Eds.), 1819-1872, North-Holland, Amsterdam, 1995.

[4] P. V. M. Blagojević, F. Frick and G. M. Ziegler, Barycenters of polytope Skeleta and counterexamples to the topological Tverberg conjecture, via constraints, (2015) 6 pages, arXiv:1508.02349

[5] P. V. M. Blagojević, B. Matschke, G. M. Ziegler, Optimal bounds for the colored Tverberg problem, J. European Math. Soc. 17 (2015) 739-754.

[6] A. Dold, Simple proofs of some Borsuk-Ulam results, Contemp. Math. 19(1983) 65-69.

[7] F. Frick, Counterexamples to the topological Tverberg conjecture, (2015), 3 pages arXiv:1502.00947

[8] J. Friedman and P. Hanlon, On the Betti numbers of chessboard complexes, J. Algebraic Combin. 8 (1998) 193-203.

[9] P. Garst, Cohen-Macaulay complexes and group actions, Ph.D.Thesis, The University of Wisconsin - Madison, 1979.

[10] I. Mabillard and U. Wagner, Eliminating higher-multiplicity intersections, I. A Whitney trick for Tverberg-type problems, (2015), 46 pages, arXiv:1508.02349

[11] J. Matoušek, Using the Borsuk-Ulam theorem, Springer-Verlag, Berlin, 2003. 
[12] J. Matoušek, Lectures on discrete geometry, Springer-Verlag, New York, 2002.

[13] M.Özaydin, Equivariant maps for the symmetric group, 1987. Available at http://minds.wisconsin.edu/handle/1793/63829

[14] T. Schöneborn and G. M. Ziegler, The topological Tverberg theorem and winding numbers, J. Combin. Theory Ser. A 112(2005) 82-104.

[15] H. Tverberg, A generalization of Radon's theorem. J. London Math. Soc. 41 (1966), 123128.

[16] R. Živaljević, S. Vrećica, The colored Tverberg's problem and complexes of injective functions, J. Combin. Theory Ser. A 61(1992) 309-318.

Authors' addresses:

Imre Bárány

Rényi Institute, Hungarian Academy of Sciences

POB 127, 1364 Budapest, Hungary

and

Department of Mathematics, University College London

Gower Street, London, WC1E 6BT, UK

E-mail: barany@renyi.hu

Gil Kalai

Einstein Institute of Mathematics, Hebrew University

Jerusalem 9190, Israel

E-mail: kalai@math.huji.ac.il

Roy Meshulam

Department of Mathematics, Technion

Haifa 32000, Israel

E-mail: meshulam@math.technion.ac.il 